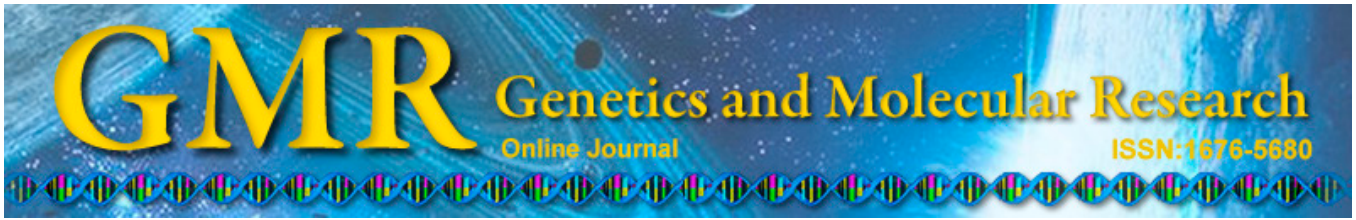

\title{
Construction and expression of prokaryotic expression vectors fused with genes of Magnaporthe oryzae effector proteins and mCherry
}

Y.Q. Yang*, H. Wang*, M.L. Liang, J.L. Yan, L. Liu, C.Y. Li and J. Yang

Key Laboratory for Agricultural Biodiversity and Pest Management of China Education Ministry, Yunnan Agricultural University, Heilongtan, Kunming, Yunnan, China

*These authors contributed equally to this study.

Corresponding author: J. Yang

E-mail: yangjin-18@163.com

Genet. Mol. Res. 14 (3): 10827-10836 (2015)

Received February 23, 2015

Accepted June 8, 2015

Published September 9, 2015

DOI http://dx.doi.org/10.4238/2015.September.9.21

\begin{abstract}
The aim of the current study was to investigate the prokaryotic expression of the Magnaporthe oryzae effector genes $B A S 1$ and BAS4 fused to the fluorescent protein mCherry. Based on previous polymorphic analysis of $B A S 1$ and $B A S 4$ in rice blast strains using PCR, blast strains containing the PCR products of $B A S 1$ and $B A S 4$ were selected for liquid culture for total RNA extraction. For PCR analysis, cDNA was selected as a template to amplify the coding region of $B A S 1$ and $B A S 4$, the plasmid pXY201 was selected as template to amplify the mCherry sequence, and the three sequences were cloned into $\mathrm{pMD}^{\circledR} 19-\mathrm{T}$ vectors. Positive recombinant plasmids were digested using two restriction enzymes and the cleaved fragments of BAS1 and mCherry and BAS4 and mCherry were ligated to pGEX4T-1 vectors and expression was induced using IPTG. The PCR results
\end{abstract}


showed that the sequence sizes of $B A S 1, B A S 4$, and mCherry were 348 , 309 , and $711 \mathrm{bp}$, respectively, and these were cloned into $\mathrm{pMD}^{\circledR} 19-\mathrm{T}$ vectors. After digestion and gel purification, the fragments of $B A S 1$ and mCherry, BAS4 and mCherry were ligated into pGEX-4T-1 vectors and expressed in Escherichia coli BL21 competent cells. The expressed proteins were approximately $60 \mathrm{kDa}$, corresponding to their theoretical size. Prokaryotic expression products of $B A S 1$ and $B A S 4$ fused to mCherry were presented in this study, providing a base for constructing prokaryotic expression vectors of pathogen effector genes fused to mCherry, which will contribute to further study of the subcellular localization, function, and protein interactions of these effectors.

Key words: Magnaporthe oryzae; Prokaryotic expression; Effector

\section{INTRODUCTION}

Rice is one of the three main food crops consumed worldwide and it constitutes the main food source of nearly half the world's population (von Braun, 2007). Rice blast is caused by an infection with the fungus Magnaporthe oryzae (Ascomycota) and it is one of the main limiting factors of high rice yield. In the 1990s, rice blast accounted for a $35 \%$ loss of rice yield worldwide every year (Oerke and Dehne, 2004). Complete sequencing of the rice and $M$. oryzae genomes has enabled gene prediction analysis using computers, but the biological function of most of the predicted genes is still unknown (Dean et al., 2005). Because many effector protein genes promote pathogenic bacteria colonization in the host, studying the function of these genes during disease occurrence and development is necessary to further explain the mechanism of interactions between M. oryzae and the host plant.

Effector proteins are pathogenic bacteria gene products that can be recognized by the host during the interaction process (Hogenhout et al., 2009). Many typical small proteins have been identified as signal peptides and genes of these are not homologous to those in any other organisms (Kamuon, 2007; Hogenhout et al., 2009). To date, 15 effector proteins have been identified, most of which are species specific, including 9 Avr proteins (PWL1, PWL2, Avr-Pita, Avr-Piz-t, Avr-Pia, Avr-Pii, Avr-Pik/km/kp, Avr-CO39, and ACE1) and the 6 most recently identified ones: BAS1, BAS2, BAS3, BAS4, S1p1, and MC69 (De Wit et al., 2009; Oliva et al., 2010). AVR-Pita is distributed widely in the protein complex and has high levels of polymorphisms caused by fragment deletion, point mutation, and transposition insertion (Zhou et al., 2007; Jia et al., 2009). AVR-CO39 only exists in the Magnaporthe grisea strain and has not been detected in the M. oryzae strain, which can infect rice. The pathogenicity towards weeping lovegrass $(\mathrm{Pwl})$ gene family encodes $P W L$, and these genes encode small secretory proteins rich in glycine, which play roles in infecting weeping lovegrass and longshan millet but are not involved in M. oryzae strain infection of rice (van der Does and Rep, 2007). The avirulence gene, $A C E 1$, a heterozyme with polyketone synthetase and nonribosomal peptide synthetase activity (Bohnert et al., 2004), is involved in the biosynthesis of a secondary metabolite with a toxicity function. The $A C E 1$ gene is expressed during appressorium formation but expression is not regulated by the signal pathway related to appressorium formation (Fudal et al., 2007). Secreted LysM Protein 1, S1p1, interferes with the chitin receptor protein 
induced signal pathway in rice to inhibit PTI (PAMPs-triggered immunity) (Mentlak et al., 2012). MC69, a novel secretory protein with a signal peptide consisting of 54 amino acids, is necessary for M. oryzae to infect rice. However, it is still unknown whether MC69 interferes with PTI or ETI in plants (Saitoh et al., 2012).

By re-sequencing the M. oryzae strain Ina168 and using PCR polymorphism analysis, three new effector proteins, Pex22, Pex31, and Pex33, consisting of approximately 100 amino acids were discovered by Yoshida et al. (2009). BAS1 to BAS4 are secretory proteins related to fungi biotrophic parasitism (Mosquera et al., 2009) in which BAS4 exists in the extracellular space in rice, while BAS1 enters the cytoplasm after secretion by M. oryzae mycelia (Khang et al., 2010).

As a result of $M$. oryzae genome sequencing, more effector protein genes have been identified. To better understand and predict the function and subcellular localization of effector protein genes, it is necessary to conduct vector construction for related effector proteins whose functions have been identified, to provide a reference and basis for identification of predicted functions of novel effector proteins. In the current study, to obtain prokaryotic expression proteins for developing a series of experiments, prokaryotic expression vectors were constructed after the apoplast effector protein gene $B A S 4$ and the cytoplasmic effector protein gene $B A S 1$ of $M$. oryzae were fused with the fluorescent protein mCherry. The results of the study provide an important reference and base for further studies of the function, subcellular localization, and protein interactions of newly discovered effector protein genes.

\section{MATERIAL AND METHODS}

\section{Vector and strain}

The cloning vector $\mathrm{pMD}^{\circledR} 19-\mathrm{T}$, DNA extraction kits, and plasmid extraction kits were purchased from TaKaRa Biotechnology (Dalian) Co., Ltd., China. The prokaryotic expression vector pGEX-4T-1 was preserved by the authors' laboratory and the binary expression plasmid was contributed by the State Key Laboratory of Crop Stress Biology for Arid Areas, NWAFU.

The results of PCR amplification indicate that the target fragments BAS1 and BAS4 exist in the M. oryzae strain A3467-40 used in the experiment. DH5 $\alpha$ and BL21 competent cells were developed by the authors' laboratory.

\section{Reagents}

Taq DNA polymerase, the DL2000 marker, and Ex Taq enzyme were purchased from TaKaRa Biotechnology Co., Ltd. China. The gel extraction kits, plasmid extraction kits, reverse transcription kits, and restriction endonucleases were purchased from Sangon Biotech (Shanghai) Co., Ltd. The TRIZOL extraction kits and reverse transcription kits were purchased from Invitrogen (Shanghai) Co., Ltd. China.

\section{Instruments}

A 9700 PCR machine (ABI), a HR410 image acquisition and analysis system (UVP), and a DYY-7C electrophoresis machine (Beijing Liuyi Biotechnology Co., Ltd. Beijing, China) were used in the experiments. 


\section{Mediums}

Antibiotics (chloramphenicol, ampicillin, rifampicin, and kanamycin), tryptone, yeast extract, and agar powder were purchased from Sangon Biotech Co., Ltd. China.

\section{Mycelia culture of the M. oryzae strain, extraction of total mycelia RNA, and reverse transcription}

Based on the results of PCR polymorphism analysis of BAS1 and BAS4 in the M. oryzae strain, mycelia masses were selected, transferred to PDA liquid medium, and cultured for 5 days, after which strains with a PCR amplification objective band were selected for activation and culturing in potato medium for 7 days. Mycelia were then collected using a sterilization filter, and total mycelia RNA was extracted using the TRIZOL extraction kits and reverse-transcribed into cDNA.

\section{Primer design and PCR amplification}

Primers were designed based on the nucleotide sequences of the $B A S 1$ and $B A S 4$ genes: BAS1-F: 5'-GCCGGATCCATGCACGTTTTCAA-3' and BAS1-R: 5'-GCCAAGCTTCGGG TAATAATTCT-3', BAS4-F: 5'-GCCGGATCCATGCAGCTCTCATT-3', and BAS4-R: 5'-GC CAAGCTTAGCAGGGGGGATAG-3', introducing BamHI and HindIII restriction sites (underlined) at both ends of the two pairs. Primers for mCherry were designed based on the nucleotide sequence of mCherry: mCherry-F: 5'-GCCAAGCTTATGGTGAGCAAGGG-3' and mCherry-R: 5'-GCCCTCGAGTCACTTGTACAGTT-3', introducing HindIII and XhoI restriction sites (underlined) at both ends. Using cDNA reverse transcribed from the mycelia of M. oryzae strain A3467-40 as a template, BAS1 and BAS4 genes were obtained by amplifying the primers BAS1-F/BAS1-R and BAS4-F/BAS4-R. Using the binary expression plasmid pXY201 as a template, $m$ Cherry genes were obtained by amplifying the mCherry primer pair. Target fragments of $B A S 1, B A S 4$, and $m$ Cherry genes were obtained from PCR products recovered from the gel slices following $1.0 \%$ agarose gel electrophoresis.

\section{Cloning the $B A S 1$ and $B A S 4$ genes}

DNA fragments obtained using gel recovery were connected to a $\mathrm{pMD}^{\circledR} 19-\mathrm{T}$ vector, transformed to Escherichia coli DH5 $\alpha$ competent cells, and cultured at $37^{\circ} \mathrm{C}$ in a constanttemperature incubator on LB plates with added ampicillin $(50 \mu \mathrm{g} / \mathrm{mL}$ and X-gal) overnight, then screened using blue-white spot screening to select white mycelia colonies for PCR identification. Positively identified clones were sent to Invitrogen Co., Ltd. for sequencing. The sequences were compared using the BioEdit software and analyzed to obtain positive clones with the correct sequence.

\section{Construction of the prokaryotic expression vector}

Cloned plasmids with the correct sequence were extracted by digesting the component cells with the restriction endonucleases Bam HI and HindIII, and the target fragments were 
isolated from the digestion products using gel recovery and purification. The prokaryotic expression vector pGEX-4T-1 was simultaneously digested using the restriction endonucleases enzymes of BamHI and XhoI, and the target fragment was obtained using gel recovery and purification. After being transformed to E. coli $\mathrm{DH} 5 \alpha$ competent cells, the ligation products of BAS1 and mCherry, BAS4 and mCherry, and pGEX-4T-1 were double digested using T4 DNA ligase at $4^{\circ} \mathrm{C}$ overnight and products were then cultured overnight on LB plates with ampicillin $(50 \mu \mathrm{g} / \mathrm{mL})$ to select transformants for PCR screening. Positive transformants were selected and cultured overnight in LB medium in order to extract plasmids and identify correct recombinants by digestion with $B a m \mathrm{HI}$ and $X h o$ I. Following identification of the correct plasmids using digestion, these were sent to Invitrogen Co., Ltd. for sequencing, and plasmids with the correct sequence were transformed to BL21 competent cells of the prokaryotic expression host bacterium to produce the prokaryotic expression product.

\section{Induced expression of target genes and purification}

Single positive colonies with the correct sequence were inoculated in $20 \mathrm{~mL}$ LB liquid medium (containing chloramphenicol: $0.033 \mathrm{mg} / \mathrm{mL}$ and ampicillin: $0.050 \mathrm{mg} / \mathrm{mL}$ ), and cultured on a shaking table at $37^{\circ} \mathrm{C}$ with a rotating speed of $170 \mathrm{rpm}$ until OD600 reached 0.5 . Using a ratio of $1: 100,5 \mathrm{~mL}$ culture suspension was extracted and inoculated in $500 \mathrm{~mL}$ LB medium containing chloramphenicol $(0.033 \mathrm{mg} / \mathrm{mL})$ and ampicillin $(0.05 \mathrm{mg} / \mathrm{mL})$, and cultured on a shaking table at $37^{\circ} \mathrm{C}$ with a rotating speed of $170 \mathrm{rpm}$, and OD600 was tested every hour. When OD600 reached 0.4 to $0.8,1 \mathrm{~mL}$ culture suspension was extracted as a control before induction, and then $500 \mu \mathrm{L}$ isopropyl- $\beta$-D-thiogalactoside, isopropylthio- $\beta$-Dgalactoside (IPTG; $100 \mathrm{mg} / \mathrm{mL}$ mother liquid) was added in $500 \mathrm{~mL} \mathrm{LB}$ liquid medium for induced culture. After $6 \mathrm{~h}$, the culture suspension was collected to extract proteins.

Collected bacteria were fully suspended in $60 \mathrm{~mL}$ 1X sodium phosphate buffer (PBS) and frozen at $-80^{\circ} \mathrm{C}$. When required, samples were removed from the freezer and crushed using an ultrasonic cell crusher after repeated freezing and thawing 3 times until the solution reached clarification. The suspension was transferred to a $50 \mathrm{~mL}$ centrifuge tube and centrifuged at $4^{\circ} \mathrm{C}, 17,500 \mathrm{rpm}$ for $50 \mathrm{~min}$. The supernatant was transferred to a newly sterilized $50 \mathrm{~mL}$ centrifuge tube and placed at $4^{\circ} \mathrm{C}$ for purification. The GST (glutathione S transferase) fusion expression product was purified using a $5 \mathrm{~mL}$ GSTrap ${ }^{\mathrm{TM}} \mathrm{FF}$ purification column (GE Amersham Biosciences).

\section{RESULTS}

\section{Amplification of M. oryzae BAS1, BAS4, and mCherry genes}

Using the BAS1-F/BAS1-R and BAS4-F/BAS4-R primer pairs and cDNA reverse transcribed from the total mycelia RNA of M. oryzae strain A3497-40 as a template, the target genes of $B A S 1$ and $B A S 4$ were obtained by PCR amplification. Using the binary expression plasmid pXY201 as a template, the mCherry gene was amplified using PCR and the designed mCherry-F/mCherry-R primer pair. Following $1.0 \%$ agarose gel electrophoresis, the target fragments of $B A S 1, B A S 4$, and mCherry were shown to be $348 \mathrm{bp}, 309 \mathrm{bp}$, and $711 \mathrm{bp}$, respectively, which are almost identical to the theoretical values, shown in Figure 1. 


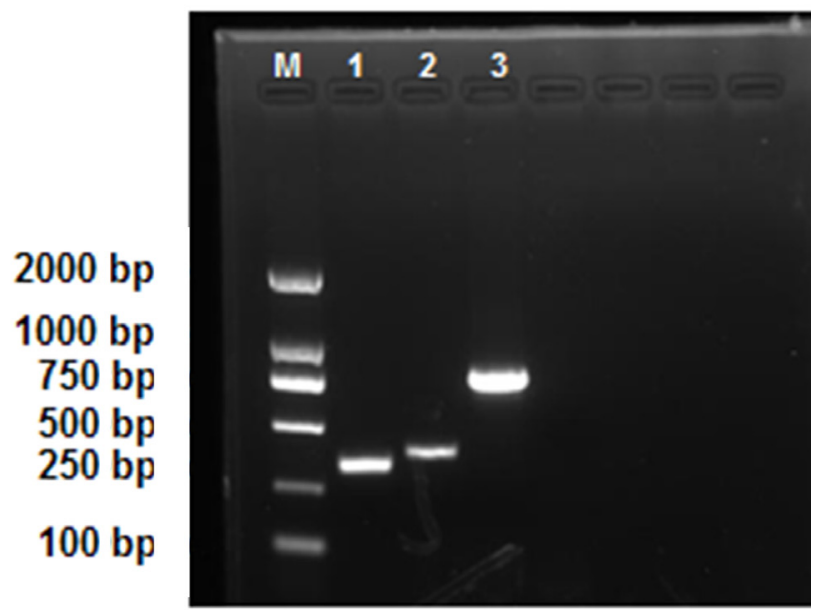

Figure 1. Amplification of Magnaporthe oryzae BAS1 and BAS4, and the strawberry red fluorescent protein gene $m$ Cherry. Lane $M=$ molecular weight standard DL2000; lanes 1 to $3=$ genes BAS1, BAS4 and $m$ Cherry, with sizes of 348,309 , and $711 \mathrm{bp}$, respectively.

\section{Cloning and identification of $B A S 1, B A S 4$, and $m$ Cherry genes}

Target gene fragments of $B A S 1, B A S 4$, and $m$ Cherry were fused to the $\mathrm{pMD}^{\circledR} 19-\mathrm{T}$ vector and transformed to DH5 $\alpha$ competent cells. Positive transformants were screened by PCR and sequenced, and those transformants with the correct sequence were cultured to extract plasmids. The recombinant plasmids pMD19-T-BAS1 and pMD19-T-BAS4 were identified by digestion using BamHI and HindIII and the recombinant plasmid pMD19-T-mCherry was identified by digestion with HindIII and XhoI, shown in Figure 2.

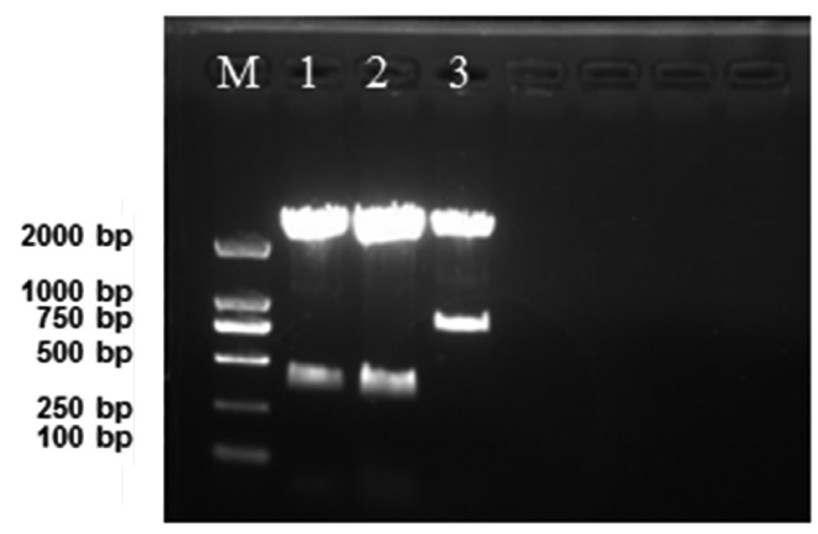

Figure 2. Identification of recombinant plasmids of Magnaporthe oryzae BAS1 and BAS4, and the strawberry red fluorescent protein gene $m$ Cherry, following double digestion. Lane $M=$ molecular weight standard DL2000; lanes 1 and 2 = digestion of recombinant plasmids pMD19-T-BAS1 and pMD19-T-BAS4 with BamHI and HindII; lane $3=$ digestion of recombinant plasmid pMD19-T-mCherry with HindIII and XhoI. 


\section{Construction and identification of prokaryotic expression vectors of $B A S 1, B A S 4$, and $m$ Cherry genes}

After plasmids pMD19-T-BAS1, pMD19-T-BAS4, and pMD19-T- $m$ Cherry were recovered using double digestion, fragments of BAS1 and mCherry, and BAS4 and mCherry were connected to the prokaryotic expression vector pGEX-4T-1, digested with BamHI and XhoI, and then transformed to the competent cells of DH5 $\alpha$. Positive transformants were screened using PCR and plasmids were extracted. Identification of recombinant plasmids using digestion with $B a m \mathrm{HI}$ and $X h o \mathrm{I}$ confirmed that the sizes of the inserted fragments were identical to the theoretical values, indicating that the target fragments had been correctly connected with the prokaryotic expression vector pGEX-4T-1 (Figure 3). By re-sequencing plasmids from the correct double digestion, recombinant plasmids with the correct sequence for pGEX-4T-1-BAS1-mCherry and pGEX-4T-1-BAS4-mCherry were obtained.

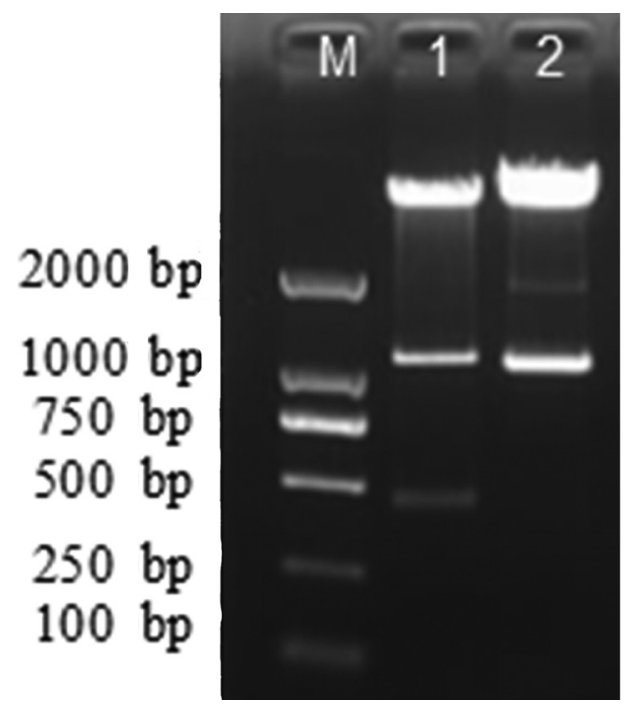

Figure 3. Identification of recombinant plasmids of Magnaporthe oryzae BAS1 and BAS4 fused to the strawberry red fluorescent protein gene $m$ Cherry, following double digestion. Lane $M=$ molecular weight standard DL2000; lanes 1 and 2 = digestion of recombinant plasmids pGEX-4T-BAS1-mCherry and pGEX-4T-BAS4- $m$ Cherry with Bam HI and XhoI.

\section{Induced expression and purification of proteins fused with $B A S 1, B A S 4$, and mCherry}

After induction by IPTG at $25^{\circ} \mathrm{C}$ for $6 \mathrm{~h}$, SDS-PAGE detection of the correct recombinant plasmids pGEX-4T-1-BAS1-mCherry and pGEX-4T-1-BAS4- $m$ Cherry transformed to the $E$. coli strain BL21 showed that the molecular weight of the protein by induced expression was approximately $60 \mathrm{kDa}$ (Figure 4), which is identical to that of the theoretical purpose fusion proteins GST-BAS1-mCherry and GST-BAS4-mCherry (molecular weight of GST tag protein is approximately $26 \mathrm{kDa}$, molecular weight of BAS1 and BAS4 proteins is approximately $10 \mathrm{kDa}$, and molecular weight of mCherry protein is approximately $28 \mathrm{kDa}$ ). 

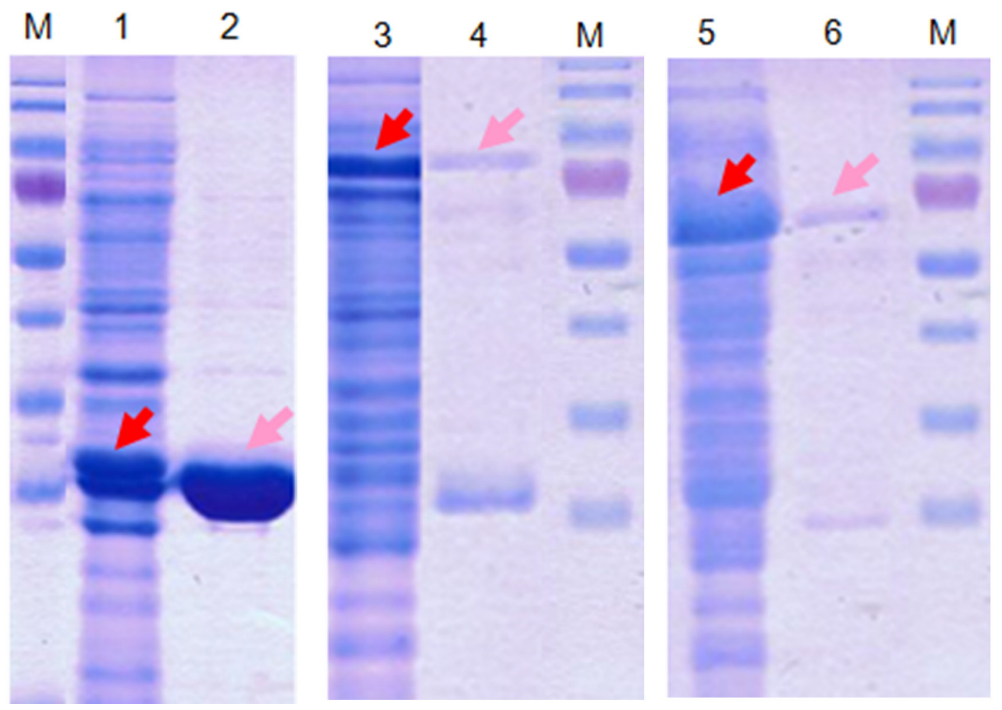

Figure 4. Expression of Magnaporthe oryzae BAS1 and BAS4 plasmids pGEX-4T-1-BAS1-mCherry and pGEX4T-1-BAS4- $m$ Cherry and purified products of SDS-PAGE. Lane $M=$ molecular weight standard of protein [170, 130, 95, 72 (red), 55, 43, 34, 26, 17, $11 \mathrm{kDa}$ ]; lane 1 = product of empty vector induced by ITPG; lane 2 = purified product of induced empty vector; lane 3 = product of pGEX-4T-1-BAS1-mCherry induced by IPTG; lane $4=$ purified product of induced pGEX-4T-1-BAS1- $m$ Cherry; lane 5 = product of pGEX-4T-1-BAS4- $m$ Cherry induced by IPTG; lane 6 = purified product of induced pGEX-4T-1-BAS4- $m$ Cherry; Red arrows indicate induced product and light pink arrows indicate purified product.

Fusion protein samples of large induced expressions were processed with an ultrasonic crusher, and fusion proteins GST-BAS1- $m$ Cherry and GST-BAS4- $m$ Cherry were purified with GST purification columns. Purified fusion proteins were collected for the following experiments. Results of the SDS-PAGE (Figure 4) show that the molecular weights of the purified fusion proteins are identical to the expected molecular weights, which indicates that the purified fusion proteins are the target proteins.

\section{DISCUSSION}

M. oryzae is a hemi-biotrophic pathogenic fungi, uptaking nutrients from the living cells of its host. Hemi-biotrophic pathogen is adopted in compatible interactions between $M$. oryzae and rice, where biotrophic pathogen is utilized when invading host cells and necrotrophic are used later (Mosquera et al., 2009). Although specialized mycelia (or infected mycelia) secret effector proteins into host cells to promote $M$. oryzae colonization of the host, the functions of these effector proteins have not been fully identified. According to their positions in the plant cell, effector proteins of M. oryzae can be divided into two types (Giraldo et al., 2013): cytoplasmic effector proteins, which enter the plant cell after being secreted, such as Avr-Pita, Pwl1, Pwl2, BAS1, and Avr-Pizt, and apoplast effector proteins, which exist in the extracellular space and do not enter the plant cell after being secreted, such as BAS4, Avr1-CO39, and Slp1. This finding shows that the effector proteins of pathogenic fungi have different functions based on their different positions after entering the host. 
Thus, confirming the localization of an effector protein can provide important theoretical knowledge for further study of its function and interaction with rice proteins. Based on the knowledge that M. oryzae effector proteins BAS1 and BAS4 play a role in rice cells following secretion by mycelia, in this paper, we cloned and constructed prokaryotic expression vectors fused with BAS1, BAS4 and mCherry, with the aim of providing a reference and basis for the research team to clone novel $M$. oryzae effector protein genes and study their secretion, localization, and function in the future.

BAS1 to BAS4 have been demonstrated to be secretory proteins related to fungal infection (Mosquera et al., 2009). Compared with PWL2 (2 cysteines) and BAS1 (0 cysteine), BAS4 is rich in cysteines (8), a key characteristic of apoplast effector proteins (Kamuon, 2007). This provides a theoretical basis upon which to analyze whether new pathogenic fungal effector proteins are cytoplasmic effector proteins or apoplast effector proteins. Movement of effector proteins in cells depends on the molecular weight of the protein, and the theoretical molecular weight of BAS1 and BAS4 proteins is small, approximately $10 \mathrm{kDa}$. For this reason, BAS1 and BAS4 were selected as reference proteins for further study of the localization and function of novel M. oryzae effector proteins.

A vector with a GST fusion tag was chosen to construct the prokaryotic expression vector because the GST tag protein itself is a highly soluble protein, which can be used to increase the solubility of heterologous proteins and it can be largely expressed in E. coli to increase expression quantity. A GST tag is also helpful for protecting recombinant proteins from degradation by extracellular proteases and to increase their stability. GST tag proteins can be eluted in mild, non-denaturing conditions to retain the protein's biological activity, thus, a vector with a GST tag was chosen in the study.

Larger quantities of protein were induced by IPTG compared with only small amounts of fusion proteins obtained from purification, which may be because crude protein flows faster through the GST purification column, or some other factors, which require further analysis. Although the quantity of fusion protein obtained by purification is smaller in the current study, the technique of constructing prokaryotic expression vectors fused with effector protein genes and mCherry and expression of soluble proteins is feasible, while the technique of GST fusion protein purification requires improvements.

To study the localization and function of effector proteins during the interaction between pathogenic bacteria and host plants, effector proteins need to be fused to fluorescent proteins in order to observe the position of proteins entering host plant cells following secretion by infected mycelia, and their subsequent position in cells. Auto-fluorescence of plants is strong and overlaps considerably with GFP, which makes fluorescence imaging observations more difficult. Therefore, GFP proteins should be avoided as much as possible when studying the interaction between pathogenic bacteria and host plants. To avoid interference from the auto-fluorescence of plants when observing the position of fluorescent proteins in rice cells in the current study, the fluorescent protein mCherry was used instead of GFP. In addition to mCherry, fluorescent proteins such as YFP and CFP can also be utilized to study the position and function of effector proteins during the interaction between rice and $M$. oryzae. Prokaryotic expression vectors, construction methods, and prokaryotic expression methods adopted in the current study provide a good reference for the research team to construct prokaryotic expression vectors of other novel M. oryzae effector proteins. 


\section{Conflicts of interest}

The authors declare no conflict of interest.

\section{ACKNOWLEDGMENTS}

Research supported by National the Natural Science Foundation of China (\#31400073) and the Yunnan Natural Science Foundation (Grant \#2013FB039) from the Yunnan Science and Technology Department of China.

\section{REFERENCES}

Bohnert HU, Fudal I, Dioh W, Tharreau D, et al. (2004). A putative polyketide synthase/peptide synthetase from Magnaporthe girsea signals pathogen attack to resistant rice. Plant Cell 16: 2499-2513.

De Wit PJ, Mehrabi R, Van den Burg HA and Stergiopoulos I (2009). Fungal effector proteins: past, present and future. Mol. Plant Pathol. 10: 735-747.

Dean RA, Talbot NJ, Ebbole DJ, Farman ML, et al. (2005). The genome sequence of the rice blast fungus Magnaporthe grisea. Nature 434: 980-986.

Fudal I, Ross S, Gout L, Blaise F, et al. (2007). Heterochromatin-like regions as ecological niches for avirulence genes in the Leptosphaeria maculans genome: map-based cloning of AvrLm6. Mol. Plant-Microbe Interact. 20: 459-470.

Giraldo MC, Dagdas YF, Gupta YK, Mentlak TA, et al. (2013). Two distinct secretion systems facilitate tissue invasion by the rice blast fungus Magnaporthe oryzae. Nat. Commun. 4: 1996.

Hogenhout SA, Van der Hoorn RA, Terauchi R and Kamoun S (2009). Emerging concepts in effector biology of plantassociated organisms. Mol. Plant-Microbe Interact. 22: 115-122.

Jia Y, Wang X, Costanzo S and Lee S (2009). Understanding the co-evolution of the rice blast resistance gene PI-TA and Magnaporthe oryzae avirulence gene AVR-PITA. In: Advances in genetics, genomics and control of rice blast disease (Wang GL and Valent B, eds.). Springer, Netherlands, 137-147.

Kamuon S (2007). Groovy times: filamentous pathogen effectors revealed. Curr. Opin. Plant Biol. 10: 358-365.

Khang CH, Berruyer R, Giraldo MC, Kankanala P, et al. (2010). Translocation of Magnaporthe oryzae effectors into rice cells and their subsequent cell-to-cell movement. Plant Cell 22: 1388-1403.

Mentlak TA, Kombrink A, Shinya T, Ryder LS, et al. (2012). Effector-mediated suppression of chitin-triggered immunity by Magnaporthe oryzae is necessary for rice blast disease. Plant Cell 24: 322-335.

Mosquera G, Giraldo MC, Khang CH, Coughlan S, et al. (2009). Interaction transcriptome analysis identifies Magnaporthe oryzae BAS1-4 as biotrophy-associated secreted proteins in rice blast disease. Plant Cell 21: 1273-1290.

Oerke EC and Dehne HW (2004). Safeguarding production-losses in major crops and the role of crop protection. Crop Prot. 23: 275-285.

Oliva R, Win J, Raffaele S, Boutemy L, et al. (2010). Recent developments in effector biology of filamentous plant pathogens. Cell Microbiol. 12: 705-715.

Saitoh H, Fujisawa S, Mitsuoka C, Ito A, et al. (2012). Large-scale gene distribution in Magnaporthe oryzae identifies MC69, a secreted protein required for infection by monocot and dicot fungal pathogens. PLoS Pathog. 8: e1002711.

van der Does HC and Rep M (2007). Virulence genes and the evolution of host specificity in plant-pathogenic fungi. Mol. Plant-Microbe Interact 20: 1175-1182.

von Braun J (2007). The world food situation: new driving forces and required actions. Food policy report. International Food Policy Research Institute, Washington.

Yoshida K, Saitoh H, Fujisawa S, Kanzaki H, et al. (2009). Association genetics reveals three novel avirulence genes from the rice blast fungal pathogen Magnaporthe oryzae. Plant Cell 21: 1573-1591.

Zhou E, Jia Y, Singh P, Correll JC, et al. (2007). Instability of the Magnaporthe oryzae avirulence gene AVR-Pita alters virulence. Fungal Genet. Biol. 44: 1024-1034. 